\title{
Manuel Edmundo Tantaleán Vidaurre
}

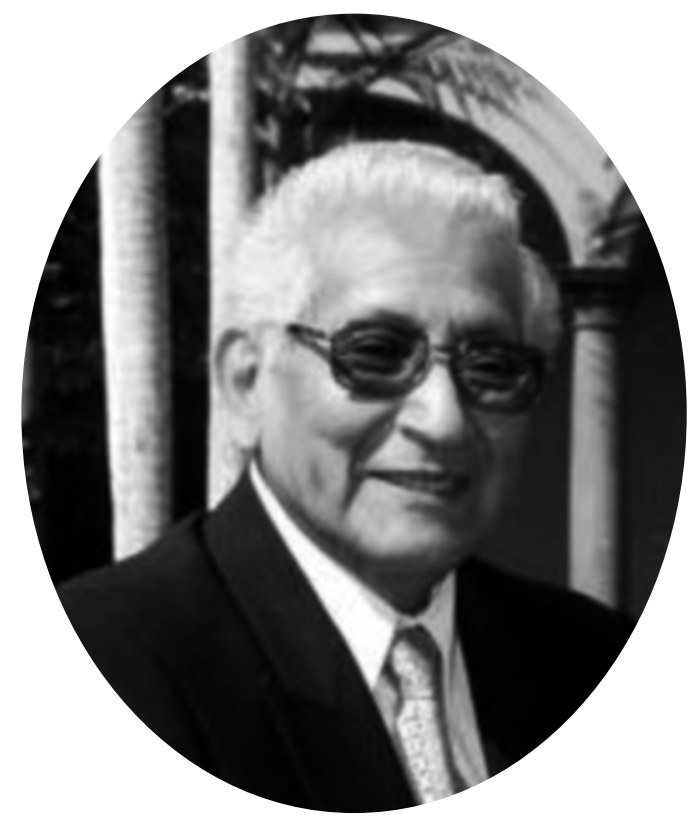

Las siguientes líneas son un homenaje al doctor Manuel Tantaleán, un gran académico, maestro y ser humano.

Manuel Edmundo Tantaleán Vidaurre nació en el Callao el 16 de noviembre de 1942. Realizó sus estudios secundarios en el colegio "2 de Mayo" y el grado de Bachiller lo obtuvo en la Facultad de Ciencias Biológicas de la Universidad Nacional Mayor de San Marcos en el año 1964. El grado de Doctor en Ciencias Biológicas, lo obtuvo en el año 1973 con la tesis "Nuevo género y nuevas especies de monogeneos de peces de algunas áreas de la costa peruana. Con redescripción de una especie conocida", trabajo que asesoró su maestra la doctora Luz Sarmiento.

El doctor Tantaleán es un académico de gran prestigio y brillante trayectoria. Ha dedicado su labor académica al estudio de los parásitos abarcando la parasitología médica, animal, marina, y de animales de vida silvestre, pero su área de mayor interés fue la helmintología. Sus trabajos sobre helmintos parásitos de peces fueron pioneros en el Perú, especialmente, los helmintos que pueden infectar al hombre.

El ha diseminado sus conocimientos e investigaciones desde el año 1965, fecha en que inició el ejercicio de la docencia universitaria, con más de 30 años ininterrumpidos en la Facultad de Medicina de la Universidad Nacional Mayor de San Marcos. Posteriormente ha enseñado en la Universidad Ricardo Palma, y recientemente en la Universidad Peruana Cayetano Heredia. La calidad de sus investigaciones le ha valido el ser invitado a dictar clases y conferencias en universidades extranjeras y en la actualidad también es profesor de la Unidad de Postgrado de la Facultad de Ciencias Biológicas de la UNMSM.

Su interés académico se volcó también en la difusión de su área de investigación, participando activamente en sociedades científicas. Es así que en el año 1974, como miembro de la Asociación de Biólogos de la Universidad Nacional mayor de San Marcos, fue miembro del primer Comité Editor de la Revista Peruana de Biología, y en la actualidad es aún colabora con ésta revista como miembro del Comité Consultivo. Fue miembro del Comité Editor de la Revista Peruana de Medicina Tropical, así como editor de la Revista Biotempo publicada por la Universidad Ricardo Palma. Recientemente, también fue uno de los fundadores y editor asociado de la revista $\mathrm{Neo-}$ tropical Helminthology de la Asociación Peruana de Helmintología e Invertebrados Afines.

Ha sido distinguido como Miembro Honorario de la Sociedad Peruana de Parasitología y el año 2009 fue nombrado Profesor Emérito de la Universidad Nacional Mayor de San Marcos.

Sus cualidades le permitieron ocupar cargos como el de Jefe de la Sección Científica de Parasitología e Inmunopatología, Jefe del Laboratorio de Diagnóstico Parasitológico y Jefe del Laboratorio de Helmintología en el Instituto de Medicina Tropical Daniel A. Carrión de la UNMSM, lugar donde forjó gran parte de sus investigaciones. 
En su producción académica podemos contar varios manuales de prácticas y técnicas y por lo menos 118 publicaciones en revistas científicas nacionales y extranjeras.

Por otro lado, muy pocos conocen las cualidades artísticas del Dr. Tantaleán. Desde muy joven fue talentoso para la música clásica, paralelamente a sus estudios universitarios integró los primeros violines de la Orquesta Filarmónica de Lima (fundada en el año 1959) bajo la dirección del maestro Leopoldo La Rosa. También, formó parte del cuarteto de cuerdas Aquarius y de otros conjuntos de cámara del que fue su director. Actualmente, dirige el coro polifónico "Nabí", perteneciente a la parroquia de Los Desamparados, Lima.
Como alumnos y discípulos del doctor Tantaleán debemos mencionar como características de su persona la honestidad, la sencillez y modestia. Como Maestro destaca su generosidad, su puntualidad, energía, su carácter exigente y minucioso, sobre todo en las investigaciones, elaboración de escritos y dirección de tesis, en fin todas las características que se esperan encontrar en los maestros de la talla del Dr. Tantaleán. Los colegas Alina Huiza, Rufino Cabrera, Lily Arrojo, Asucena Naupay, Hermes Escalante y la que suscribe el presente, nos sentimos orgullosos de tener a un gran maestro y poder considerarnos sus discípulos.

\section{Rosa Martinez Rojas}

\section{Publicaciones científicas del doctor Manuel Edmundo Tantaleán Vidaurre}

La presencia de larvas de Anisakis sp. en peces comerciales del mar peruano. Revista Peruana de Medicina Tropical UNMSM. 1: $38-43 ; 1972$.

Prevalencia de enteroparásitos en los distritos de San Juan y Magdalena (Departamento de Cajamarca) 1971. Revista Peruana de Medicina Tropical UNMSM. 2: 37-41; 1971.

Monogeneos de la familia Microcotylidae Taschenberg, 1879 parásitos de peces del mar peruano con descripción de una especie nueva. Biota 10: 120-127; 1974.

Parabatrachostrongylus lumbrerasi n.g., n.sp. (Nematoda: Trichostrongylidae) parásito de anfibio de Arequipa. Biota 10: $159-163 ; 1974$.

Dos nuevas especies de Monogeneos parásitos de peces comerciales del mar peruano. Biota 10: 235-242; 1974.

Los hospederos intermediarios de Fasciola hepatica en el Perú. 1. Estudio de la infección natural y experimental de Lymnaea viator, Lymnaea diaphana y Physa venustula. Biota 10: 243-250; 1974.

Monoecocestus parcitesticulatus Rego, 1960 (Anoplocephalidae) un nuevo cestodo para el Perú. Revista Peruana de Biología 1: $81 ; 1974$

La presencia de Fasciola hepatica en la localidad de Huinco. Comprobación experimental. Revista Peruana de Biología 1: $136-146 ; 1974$

La infección de cangrejos procedentes del valle de Condebamba (Cajamarca) por metacercarias de Paragonimus. Revista Peruana de Biología 1: 192-193; 1974.

La vía de penetración de la larva de Paragonimus peruvianus en animales de experimentación. Revista do Instituto de Medicina Tropical Sao Paulo 16: 332-336; 1974.

Pseudoeurysorchis sarmientoi n.g., n.sp. (Monogenea: Diclidophoridae) parásito de pez comercial del mar peruano. Revista Brasilera de Biología 34: 253-258; 1974.

Lesiones histopatológicas en pulmón de gato doméstico inoculado experimentalmente con Paragonimus peruvianus. Revista Peruana de Medicina Tropical UNMSM. 3-4: 32-41; 1975.

Estudio de algunos tremátodos del Perú. Revista Peruana de Medicina Tropical UNMSM 3-4: 46-56; 1975.
Monogeneos de peces de las costas del Perú. I. Axine ibañezi n.sp. parásito de un pez volador (Exocoetus volitans). Revista de Biología Tropical 22: 211-215; 1975.

Hallazgo de larvas plerocercoides de Diphyllobothriidae Lühe, 1910 (Cestoda) en peces del mar peruano. Boletín Chileno de Parasitología 330: 18-20; 1975.

Contribución al conocimiento de los helmintos de vertebrados del Perú. Biota 10: 437-443; 1976.

Los hospederos intermediarios de Fasciola hepatica en el Perú. 2. Infección experimental de Lymnaea columella Say. Biota 11: 34-37; 1976.

La paragonimiasis en la población de los distritos de San Juan y Magdalena (Cajamarca) 1971. Biota 11: 23-33; 1976.

Observaciones sobre la biología del cisticercoide de Hymenolepis diminuta. Boletín Peruano de Parasitología 2: 89; 1976.

Estudio de algunos nemátodos de "cucarachas" del Perú. Boletín Peruano de Parasitología 2: 51-56; 1980.

Estudio sobre los helmintos de peces elasmobranquios de la costa peruana. 1. Nuevos registros de Tetraphyllideos. Boletín Peruano de Parasitología 2: 71-75; 1980.

Estudio de la larva de cestode parásito de Mesodesma donacium L. "macha" de la costa peruana. Boletín Peruano de Parasitología 3: 22-27; 1981.

Helmintos parásitos de peces marinos de la costa peruana. NCTL. Serie de Divulgación Científica No 1 . Lima. 40 pp. 1982.

Parásitos de primates peruanos: Helmintos del "mono fraile" y del "pichico barba blanca". Boletín de Lima 5: 54-58; 1983.

Helmintos nuevos para el Perú. Boletín de Medicina Tropical (Lima) 2: $1-3 ; 1983$.

Primer caso en el Perú de infección humana por Dirofilaria immitis. Boletín de Medicina Tropical (Lima) 2: 4-5; 1983.

Larva migrans visceral producida por Toxocara canis en ratones albinos. Boletín de Medicina Tropical (Lima) 3: 10-11; 1984.

La hidatidosis en el Perú: importancia del inmunodiagnóstico y de los estudios seroepidemiológicos. Acta Médica 11: 21-28; 1984.

La Diphyllobothriasis y su epidemiología en el Perú. 1. Los hospederos paraténicos de Diphyllobothrium pacificum. Boletín de Medicina Tropical (Lima) 3: 10-11; 1984. 
Contribución al conocimiento de la helmintofauna de peces marinos de la costa peruana. Parasitología al Día 9: 40-43; 1985.

Helmintos parásitos de peces de agua dulce del Perú. Universidad Nacional del Altiplano. Facultad de Medicina Veterinaria. Puno. 1985.

Relación huésped-parásito entre Paragonimus mexicanus y vertebrados. I. Ensayo experimental en roedores. Boletín de Medicina Tropical (Lima) 4: 22-24; 1985.

Monogeneos de las costas del Perú. II. Cambio de nombre por homonimia y nuevos registros. Revista de la Facultad de Ciencias Veterinarias Universidad Central de Venezuela 32: 91-95; 1985.

Parásitos de Bothrops atrox (Viperidae) de la amazonía peruana. Revista de la Asociación de Médicos Veterinarios Especialistas en Animales Menores 20: 11-12; 1985.

Observaciones sobre la presencia de cuatro enteroparásitos en la región de rupa-rupa en el departamento de Huánuco. Boletín de Medicina Tropical (Lima) 5: 7-9; 1986.

Paragonimus y paragonimiasis en el Perú. (1a. parte). Boletín de Medicina Tropical (Lima) 5: 33-39; 1986.

La ruta migratoria de la larva de Paragonimus mexicanus Miyazaki e Ishii, 1969 en el gato doméstico infectado experimentalmente. Revista de Ciencias UNMSM. 74: 63-69; 1986.

Presencia de Paragonimus sp. en pulmones de cerdo doméstico (Sus scrofa domestica) Pucallpa, Perú. Boletín de Medicina Tropical (Lima) 5: 54-56; 1986.

Paragonimus y paragonimiasis en el Perú. (2a. parte). Boletín de Medicina Tropical (Lima) 5: 72-80; 1986.

Nuevos registros de helmintos parásitos de peces elasmobranquios de las costas del Perú. Revista de Biología Tropical, 35: 167-168. 1987.

Nota sobre la presencia de Synthesium tursionis (Marchi, 1873) Price, 1932 (Trematoda: Campulidae) en Delphinus delphis L. (Cetacea: Odontoceti) de la costa peruana. Parasitología al Día 11: 39-40; 1987.

Cynoscionicola americana n. sp. y C. cynoscioni n. sp. (Monogenea: Heteraxinidae) parásitos de peces de la familia Sciaenidae de la costa peruana. Parasitología al Día 11: $55-61 ; 1987$

Aquaculture with tretated wastewater: a status report on studies conducted in Lima, Peru. App. Res. and Tech (WUDAT). Tech. note $\mathrm{N}^{\mathrm{o}}$ 3. The World Bank. 1987.

Algunos nematodes de Diplopoda del Perú, con descripción de una especie nueva. Biota 13: 13-19; 1987.

Gorgoderina sp. y Batrachotaenia sp. parásitos de Batrachophrynus macrostomus Peters, 1873 (amphibios) de la laguna de Huicra, Pasco. Biota 13: 34-39; 1987.

Una especie nueva y nuevos registros de platyhelmintos parásitos de peces marinos. Boletín de Lima 10: 91-96; 1988.

Cinco nematodes parásitos de "milpies" (Diplopoda, Spirostreptidae) de Amazonas-Perú. Revista Peruana de Entomología 31: 147-150; 1988 (1989).

Contribución al estudio de los helmintos parásitos de anfibios del Perú. Boletín de Lima 11: 69-77; 1989.

Notes on some helminth parasites from peruvian monkeys. Laboratory Primate Newsletter 29: 6-8; 1990.

Zoonosis parasitaria en un mono "choro" (Lagothrix lagotricha). MV. Revista de Ciencias Veterinarias 5: 19-20; 1989.

Nagmia peruviana n. sp. (Digenea: Gorgoderidae: Anaporrhutinae) un nuevo parásito de la "manta", Mobula lucasana B. y T. de la costa del Perú. Revista de Ciencias UNMSM. 75: 25-29; 1990.

Acción "in vitro" del derivado nitro vinil furánico (Sq 18506) sobre las infecciones experimentales del ratón por Trypanosoma cruzi y Fasciola hepatica. Boletín Peruano de Parasitología 4-10: 10-14; 1982-1988. (Publ. 1990).
Algunos nemátodes parásitos de equinos del departamento de Puno, Perú. Boletín Peruano de Parasitología 4-10: 38-39; 19821988. (Publ. 1990).

Presencia de Spirometra mansonoides en el Perú. Boletín Peruano de Parasitología 4-10: 46; 1982-1988. (Publ. 1990).

Nuevos helmintos parásitos de peces elasmobranquios de la costa peruana. Boletín de Lima 13: 25-28; 1991.

Stenurus australis n. sp. (Nematoda: Pseudaliidae) de Phocoena spinipinnis (Burmeister, 1865) (Cetacea: Phocoenidae) de Perú. Publicaciones del Museo de Historia Natural UNMSM. Serie A Zoología 36: 1-4; 1991.

Aporocotyle garciai n. sp. (Digenea: Sanguinicolidae) parásito de Genypterus sp. de la costa peruana. Parasitología al Día 14: 67-69; 1990 (Publ. 1991)

Dos casos de infestación humana producida por Melophagus ovinus (Diptera, Hipobosciidae). Revista Peruana de Medicina Tropical UNMSM. 5: 105; 1991.

Digeneos (Trematoda) del Perú. Boletín de Lima 14: 47-84; 1992.

Nota sobre algunos helmintos nuevos para el Perú. Revista Peruana de Medicina Tropical UNMSM. 6: 85-86; 1992.

Ascariasis en primates no humanos del Perú. Revista Peruana de Medicina Tropical UNMSM. 6: 87; 1992.

Tipos de cercaria de trematodos en algunas zonas del Perú. Biota 14: 42-57; 1992.

Nematode larvae with medical importance found in sea fish from the peruvian shore, with two records of human infections. Revista Peruana de Medicina Tropical UNMSM. 7: 61-65; 1993.

Algunos helmintos de mamíferos marinos del Perú y su importancia médica. Revista Peruana de Medicina Tropical UNMSM. 7: 67-71; 1993.

Trematodes de la familia Gorgoderidae en anfibios Leptodactylidae de la región altoandina del Perú. Boletín de Lima 15: 25-27; 1993.

Scanning and Transmission electron microscopy of the tegument of the metacercariae of Paragonimus peruvianus. Parasitología al Día 17: 5-10; 1993.

Nuevos registros de helmintos parásitos en peces marinos de la costa peruana. Memorias de X Congreso Nacional de Biología. 02-07 Agosto, Lima, Perú. pp. 293-298; 1993.

Estructura antigénica de Paragonimus mexicanus. Revista Peruana de Medicina Tropical UNMSM. 7: 81-89; 1993.

Eliminación de hidátide por la vía rectal. Revista Peruana de Medicina Tropical UNMSM. 7: 97-98; 1993.

Nota sobre parasitismo intestinal diagnosticado en el IMT "Daniel A. Carrión". Revista Peruana de Medicina Tropical UNMSM. 7: 99-103; 1993.

Ictericia posthepática trematódica en un mono "pichico barriga colorada" (Saguinus labiatus) (Primates: Callithrichidae). Revista de Investigaciones Pecuarias IVITA (Perú) 6: 124-125.

Miocardiopatía asociada a microfilarias en un mono nocturno (Aotus vociferans) (Primates: Cebidae). Revista de Investigaciones Pecuarias IVITA (Perú) 6: 126-127; 1993.

Detección de anticuerpos en gatos infectados con Paragonimus mexicanus mediante la contrainmunoelectroforesis. Revista Peruana de Medicina Tropical UNMSM. 8: 53-55; 1994.

Enzimas proteolíticas liberadas in vitro por adultos de Paragonimus mexicanus. Revista Peruana de Medicina Tropical UNMSM. 8: 57-61; 1994.

Detección de antígenos de Paragonimus en las heces de un gato con infección extrapulmonar mediante contrainmunoelectroforesis. Revista Peruana de Medicina Tropical UNMSM. 8: 63-64; 1994

Nuevos helmintos de importancia médica en el Perú. Revista Peruana de Medicina Tropical UNMSM. 8: 87-91; 1994. 
Determinación del huésped intermediario de Fasciola hepatica en la irrigación de Asillo, Azángaro, Puno. Revista Peruana de Medicina Tropical UNMSM. 8: 93-94; 1994.

Sinopsis de los parásitos de peces marinos de la costa peruana. Biotempo 1: 53-101; 1994.

Parasites of the Aotus monkey. In: AOTUS: THE OWL MONKEY. Ed.: Baer, J.F., Weller, R.E. and Kakoma, I. Academic Press Inc. San Diego. p: 353-374; 1994.

Algunos helmintos de Scomber japonicus "caballa" de la Reserva Nacional de Paracas. Biotempo 2: 85-86; 1995.

Parasitic protozoa in neotropical primates. Laboratory Primate Newsletter 35: 1-7; 1996.

Patogenicidad de las larvas de Anisakis physeteris inoculadas experimentalmente en Canis familiaris. Parasitología al Día 21: 14-19; 1997.

Cestodos parásitos de murciélagos de Ica, Perú. Parasitología al Día 21: 20-24; 1997.

Una nueva especie y un nuevo registro de monogeneos parásitos en peces marinos de las costas del Perú. Parasitología al Día 22: 41-44; 1998.

Primer registro en el Perú de Raillietina (R.) demerariensis (Cestoda: Taenioidea) en Rattus rattus norvegicus. Revista Peruana de Biología 5: 67-70; 1998.

Identificación del huésped intermediario de Fasciola hepatica en la provincia de Candarave, Tacna. Revista Peruana de Parasitología 13: 63-65; 1998

La infección de cangrejos por metacercarias de Paragonimus en el valle de Condebamba, departamento de Cajamarca. Revista Peruana de Parasitología 13: 66-71; 1998.

Nuevos registros de nemátodes parásitos de animales de vida silvestre en el Perú. Revista Peruana de Biología 5: 103$104 ; 1998$.

Algunos helmintos de la marsopa espinosa, Phocoena spinipinnis de la Reserva Nacional de Paracas, Perú. Parasitología al Día 23: 56-57; 1999.

Nemátodos parásitos del hombe y de los animales en el Perú. Revista Peruana de Parasitología, 14: 9-65; 1999.

Diphyllobothrium pacificum (Nybelin, 1931) Margolis, 1956 en Canis familiaris de la ciudad de Chincha, Perú. Boletín Chileno de Parasitología 56: 26-28; 2001.

Aspectos del comportamiento biológico de la cepa TCI de Trypanosoma cruzi. Revista Peruana de Parasitología, 15: 9-14; 2000-2001 (2002).

Lymnaea columella Say (=Pseudosuccinea columella) como huésped intermediario de Fasciola hepatica en el Perú. Revista Peruana de Parasitología, 15: 33-35; 2000-2001 (2002).

Parásitos presentes en Periplaneta americana Linnaeus "cucaracha doméstica" de la ciudad de Ica. Revista Peruana de Biología, 8: 105-113; 2001.

Observaciones experimentales sobre el xenodiagnóstico en tripanosomiasis americana. Revista Peruana de Biología, 8: $171-173 ; 2001$.

Diphyllobothrium pacificum en niños del Perú. Diagnóstico, 41: 161-164; 2002.

Profilicollis altmani (Perry, 1942) Van Cleave, 1947 (Acanthocephala) en el Perú. Con notas sobre la infección experimental de mamíferos terrestres. Revista Peruana de Biología, 9: $49-51 ; 2002$.

A survey for helminth parasites in feral New World non-human primate populations and its comparison with parasitological data from man in the region. Journal of Medical Primatology, 32: 341-345; 2003.
Hiperendemicidad de Fasciolosis humana en el valle del Mantaro: Factores de riesgo de la infección por Fasciola hepatica. Revista de Gastroenterología. Perú. 24: 158-164; 2004.

Registro de nuevo huésped intermediario de Hymenolepis diminuta (Cestoda) en el Perú. Revista peruana de Biología, 11: 107-108; 2004.

Consideraciones sobre Profilicollis altmani (Perry, 1942) Van Cleave, 1947 en el Perú. Revista Peruana de Biología, 11: 109-111; 2004.

Registro de Copiatestes filiferus (Leukart in Sars, 1885) Gibson y Bray, 1977 (Digenea) y Gorgorhynchus sp. (Acanthocephala) en pez marino de la costa peruana. Revista Peruana de Biología, 11: 223-224; 2004.

Wild animals endoparasites (Nemathelminthes and Plathelminthes) from the Manu Biosphere Reserve, Peru. Revista Peruana de Biología, 11: 219-222; 2004.

Parásitos helmintos en Boa constrictor, Epicrates cenchria y Corallus caninus (Ophidia: Boidae) criadas en autiverio. Revista de Investigaciones Veterinarias, Perú. 15: 166-169; 2004.

Fasciolosis errática. Revista Peruana de Medicina Experimental, 21: $276-279 ; 2004$

Altas tasas de prevalencia de fasciolosis humana en el Perú: una enfermedad emergente. Revista Peruana de Enfermedades Infecciosas y Tropicales, 3: 8-13, 2005.

Acantocéfalos del Perú. Revista Peruana de Biología, 12: 83-92; 2005.

Huéspedes definitivos de Spirometra mansonoides (Cestoda, Diphyllobothriidae) en el Perú. Revista Peruana de Biología, 12: 153-157; 2005.

Parásitos gastrointestinales de la taricaya, Podocnemis unifilis (Troschel, 1848) (Testudines: Podocnemididae) de Iquitos, Perú. Revista Peruana de Biología, 13: 119-120; 2006.

El zorro andino, Pseudalopex culpaeus, un nuevo huésped para Corynosoma obtuscens (Acanthocephala) en el Perú. Revista Peruana de Biología, 14: 51-52; 2007.

Presencia en el Perú de Mecistocirrus digitatus (Linstow, 1906) Railliet \& Henry, 1912 (Nematoda, Trichostrongylidae, Haemonchinae). Revista Peruana de Biología, 14: 313 $314 ; 2007$.

Prevalencia de helmintos intestinales de una población de monos maquisapas silvestres, Ateles belzebuth chamek en el Parque Nacional del Manu, Perú. Neotropical Helminthology, 2: 19-26; 2008 (Coautor).

Cervical tumor caused by the sexually mature stage of Fasciola hepatica. Transactions of the Royal Society of Tropical Medicine and Hygiene, 103: 318-320; 2009 (Coautor)

Tricholeiperia peruensis n. sp. (Nematoda, Molineidae) del quiróptero Lophostoma silvicolum occidentalis (Phyllostomidae) en Tumbes, Perú. Revista Peruana de Biología, 15-23-26; 2009 (Coautor).

Frecuencia de helmintos en huanganas silvestres (Tayassu pecari Link, 1795) residentes en áreas protegidas del departamento de Madre de Dios, Perú. Neotropical Helminthology, 2: 48-53; 2008 (Coautor). Publicado 2009.

Cestodos de quirópteros del Parque Nacional Cerros de Amotape, Tumbes, Perú. Revista Peruana de Biología, 16: 57-60, 2009 (Coautor).

Viscachataenia quadrata Denegri, Dopchiz, Elissondo \& Beveridge, 2003. (Cestoda: Anoplocephalidae) en el Perú. Revista Peruana de Biología, 16: 129-130, 2009.

Presencia de Cotylophoron cotylophorum (Trematoda: Paramphistomidae) en bovinos de Loreto, Perú. Revista Peruana de Biología, 16: 141-142, 2009 (Coautor). 